\title{
Single-molecule imaging reveals topological isomer-dependent diffusion by 4-armed star and dicyclic 8-shaped polymers
}

\author{
Satoshi Habuchi, ${ }^{*}$ Susumu Fujiwara, ${ }^{b}$ Takuya Yamamoto ${ }^{b}$ and Yasuyuki Tezuka ${ }^{*}$,
}

Diffusion dynamics of topological isomers of polymer molecules was investigated at the single-molecule level in a melt state by employing the fluorophore-incorporated 4-armed star and the corresponding doubly-cyclized, 8-shaped poly(THF) chains. While the single-molecule fluorescence imaging experiment revealed that the diffusion of the 4-armed star polymer was described by a single Gaussian distribution, the diffusion of the 8-shaped polymer exhibited a double Gaussian distribution behaviour. We reasoned that the two 8-shaped polymeric isomers have distinct diffusion modes in the melt state, although ensemble-averaged experimental methods cannot detect differences in overall conformational state of the isomers. The singlemolecule experiments suggested that one of the 8-shaped polymeric isomer, having the horizontally oriented form, causes an efficient threading with the linear matrix chains which leads to the slower diffusion compared with the corresponding 4-armed star polymer, while the other 8-shaped polymeric isomer, having the vertically oriented form, displayed faster diffusion by the suppression of effective threading with the linear matrix chains due to its contracted chain conformation.

\section{Introduction}

Diffusion and relaxation of polymer chains are the key factors in determining their rheological properties, ${ }^{1}$ which serve as a basis for the development of polymer materials such as thin films and fibres. In contrast to linear polymers whose chain dynamics have been well characterized, ${ }^{2-5}$ topological polymers such as ring and dicyclic polymers often display unexpected static and dynamic behaviours. ${ }^{6}$ For instance, it was recently demonstrated that the thermal and salt stability of the micelles formed from a polymer increased significantly by a linear-tocyclic conversion. ${ }^{7,8}$ Such topology effect should arise from the unique inter- and intra-chain interactions of cyclic polymers which are originated from the absence of chain ends. ${ }^{9}$ In addition to the topology dependent chain interactions, topological polymers often adopt different isomeric forms that do not exist in linear and branched polymers because of their unique structural configurations. Such topological isomers often display distinct physical properties such as isomerdependent hydrodynamic volume. ${ }^{10,}{ }^{11}$ This could have significant effects on their diffusive and relaxation dynamics, and thus could contribute to the development of new polymer materials through a topological isomer-dependent control of the rheological properties. However, molecular mechanisms of topology- and topological isomer-dependent diffusion and relaxation have not been well understood despite the extensive theoretical and experimental studies.

While ensemble averaged experimental methods have been the primary tools to characterize diffusive and relaxation dynamics of polymers, ${ }^{12-14}$ recent single-molecule imaging studies have demonstrated spatiotemporal heterogeneities of polymer dynamics. ${ }^{15-21}$ Importantly, those observations cannot be fully interpreted by the current polymer physics theory. Especially, given the unusual and complicated chain dynamics and interactions in topological polymers, single-molecule approaches provide a powerful means for investigating their dynamics. ${ }^{22}$ We have recently reported a topology dependent diffusion of individual linear and cyclic polymers in a semidilute solution and in a melt state by employing a linear and cyclic poly(THF)s containing a perylene diimide moiety as a fluorophore. ${ }^{23-25}$ The single-molecule imaging experiments have revealed an unexpected multiple-mode diffusion of the cyclic polymer in the semidilute solution due to partial threading of the cyclic chains with the linear matrix chains. ${ }^{23}$ The study clearly demonstrated the topology effect on the diffusion dynamics of polymers that was often hidden behind the ensemble averaging in the conventional ensemble experiments.

In the present study, we focused on the diffusive dynamics of topological isomers of polymer molecules in a melt state at the single-molecule level as this could be a critical factor for controlling polymer dynamics while only little is known about their fundamental physical properties. As a model system of topological isomers, we synthesized a pair of 4-armed star (1) and dicyclic 8 -shaped polymers. ${ }^{26}$ In order to fluorescently visualize the diffusive dynamics of the entire polymers, we have introduced a perylene diimide fluorophore unit commonly in the core junction position of the 4-armed star and dicyclic 8shaped polymer structures by an electrostatic self-assembly and covalent fixation (ESA-CF) process, which has been reported previously (Figure 1). ${ }^{10,27,} 28$ The 8-shaped polymer employed in the present study is considered as an isomeric mixture having vertically oriented (2a) and horizontally oriented (2b) forms (Figure 1(b)), as reasonably presumed from the previous studies on the double cyclization by $\mathrm{H}$-shaped polymer precursors. ${ }^{29}$ Importantly, the presence of two distinguishable polymeric components could not be substantiated unequivocally through NMR or SEC measurements. Now upon a single-molecule fluorescence imaging study on the set of 4-armed star polymer and a pair of isomeric dicyclic 8-shaped poly(THF) incorporating a perylene diimide fluorophore, we demonstrate remarkable topology and isomer effects in their dynamics, in particular undetectable through any ensemble methods (e.g. NMR, SEC). 


\section{Experimental}

\section{Sample preparation}

1, 2a, and $\mathbf{2 b}$ were synthesized using an electrostatic selfassembly and covalent fixation process, which is reported elsewhere. $^{10,27,28} \mathbf{1}$ and $\mathbf{2 a + 2 b}$ have number average molecular weights of $M_{\mathrm{n}}=8,300\left(M_{\mathrm{w}} / M_{\mathrm{n}}=1.04\right)$ and $M_{\mathrm{n}}=12,500\left(M_{\mathrm{w}} / M_{\mathrm{n}}\right.$ $=1.10)$, respectively. ${ }^{26}$ Samples for the single-molecule fluorescence imaging experiments were prepared by mixing a melt of unlabelled linear poly(THF) (Aldrich, $M_{\mathrm{n}}=3,000$, $\left.M_{\mathrm{w}} / M_{\mathrm{n}}=1.89\right)$ and a chloroform solution of $1\left(1 \times 10^{-6} \mathrm{M}\right)$ or $\mathbf{2 a}+\mathbf{2 b}\left(1 \times 10^{-6} \mathrm{M}\right)$ at the volume ratio of 99 to 1 . Then, the solvent was evaporated completely by heating the mixture. The extremely low mole fraction of $\mathbf{1}$ and $\mathbf{2 a + 2 b}$ in the melt samples $(32-35 \mathrm{ppb})$ allows spatial isolation of the fluorophore incorporated molecules that is required for the single-molecule analysis of diffusion. A $10 \mu \mathrm{L}$ volume of the sample was sandwiched between two clean microscope coverslips. The thickness of the melt sample was approximately $10 \mu \mathrm{m}$.

\section{Single-molecule fluorescence imaging experiment}

Single-molecule fluorescence images were recorded by a homebuilt imaging setup which consisted of an inverted microscope (Olympus, IX71) equipped with a high N.A. objective lens

(a)

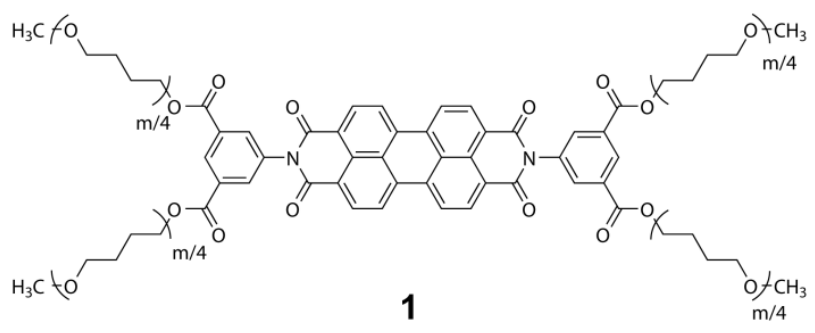

(b)

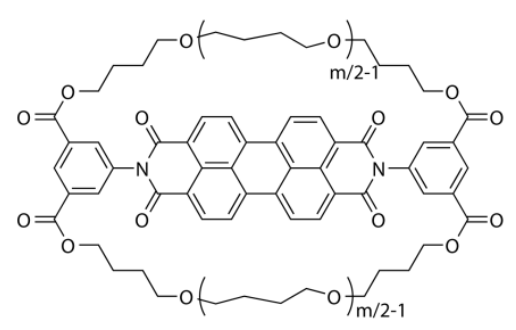

$2 \mathbf{a}$

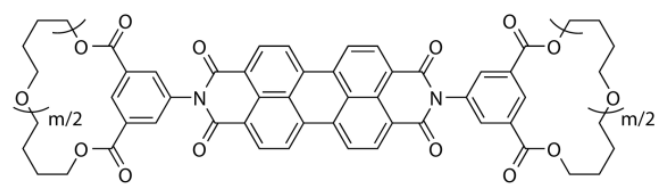

$2 \mathbf{b}$

Figure 1. The 4-armed star (1), vertically oriented 8-shaped dicyclic (2a), and horizontally oriented 8-shaped dicyclic (2b) poly(THF) molecules containing a perylene diimide moiety.
(Olympus, $\times 100$, N.A. $=1.3$ ) and EM-CCD camera (Andor Technology, iXon $\left.{ }^{\mathrm{EM}}+\right)^{23}$ The temperature of the sample was set to $303 \mathrm{~K}$ using an objective heater (Bioptechs, temperature stability: $\left.\pm 0.2{ }^{\circ} \mathrm{C}\right) .{ }^{25}$ The sample was set on the microscope stage and kept at $303 \mathrm{~K}$ for 1 hour before recording the fluorescence images. This ensures that the sample has reached the equilibrium condition. A circularly-polarized light from a $488 \mathrm{~nm}$ line of a CW Ar-Kr ion laser (Coherent Innova 70C) obtained by a Berek compensator (Newport) was used for excitation. The excitation light was introduced into the microscope through an excitation filter (Semrock, LL01-48812.5), reflected by a dichroic mirror (Omega optical, 500DRLP), and the sample was illuminated through the objective. We adjusted an illumination area to approximately $20 \mu \mathrm{m}$ in diameter. The average excitation power was $2 \mathrm{~kW} \mathrm{~cm}^{-}$ 2 . The fluorescence from the sample was corrected by the same objective, passed through the dichroic mirror and an emission filter (Semrock, BLP01-488R-25), and was detected by the EM-CCD camera. The fluorescence images were recorded at $133 \mathrm{~Hz}$ (i.e., $7.5 \mathrm{~ms}$ exposure time). The pixel size of the images was $160 \mathrm{~nm}$.

\section{Image analysis}

The diffusion rate and mode of $\mathbf{1}$ and $\mathbf{2 a}+\mathbf{2} \mathbf{b}$ were analysed by single-molecule localization and tracking method. ${ }^{23}$ First, the locations of the molecules in each image frame were precisely determined by two-dimensional Gaussian fitting using routines written in Matlab. ${ }^{30,} 31$ Then, the diffusion coefficients of individual molecules $(D)$ were determined by mean-squared displacement (MSD) analysis of the trajectories. In order to compare experimentally determined $D$ distributions with theoretically calculated probability distributions, the diffusion trajectories were all cut such that they contained ten data points (see below).

\section{Cumulative distribution function (CDF) analysis}

The modes of diffusion were analysed using a CDF, $P\left(r^{2}, i \Delta t\right)$, which corresponds to the cumulative probability of finding the diffusing molecule within a radius $r$ from the origin after a certain time lag $i \Delta t$.

Homogeneous DIFFUSION MODEL. CDFs obtained for molecules that show homogeneous diffusion with a distinct $D$ value were fitted by equation, ${ }^{32}$

$P\left(r^{2}, i \Delta t\right)=\int_{0}^{r}\left(r^{2^{\prime}}, i \Delta t\right) \mathrm{d} r^{\prime}=1-A \cdot \exp \left[-\frac{r^{2}}{4 D(i \Delta t)}\right]$

Any deviation from a single-exponential decaying behaviour suggests the heterogeneous diffusion of the molecules in the sample. The CDF coefficients $\left(r^{2}\right)$ at different time lags were determined by fitting the CDFs with equation 1 . The $D$ value was estimated by the slope of the $r^{2}$ plot.

GAUSSIAN DISTRIBUTION MODEL. CDFs obtained for molecules that show multiple diffusion modes in which the $D$ value distribution was described by a Gaussian were fitted by equation, ${ }^{25}$ 
$1-P\left(r^{2}, i \Delta t\right)=\int_{0}^{\infty} f(D) \cdot D^{-1} \cdot \exp \left[-\frac{r^{2}}{4 D(i \Delta t)}\right] \mathrm{d} D$

$f(D)=A \cdot \exp \left\{-\frac{\left(D-D_{0}\right)^{2}}{2 w^{2}}\right\}$

where $f(D)$ denotes probability distribution of $D$ described by a Gaussian. $A, w$, and $D_{0}$ are the amplitude, width, and centre of the Gaussian, and those values were determined by the fitting. The $r^{2}$ values at different time lags were determined by fitting the CDFs with equation 2 . The mean $D$ value was estimated by the slope of the $r^{2}$ plot.

Double Gaussian MODEL. CDFs obtained for molecules that show multiple diffusion modes in which the $D$ value distribution was described by multiple continuous and broad distribution (i.e., multiple Gaussian) were fitted by equation,

$1-P\left(r^{2}, i \Delta t\right)=\sum A_{j} \cdot \int_{0}^{\infty} f\left(D_{j}\right) \cdot D_{j}^{-1} \cdot \exp \left[-\frac{r^{2}}{4 D_{j}(i \Delta t)}\right] \mathrm{d} D_{j}$

$f\left(D_{j}\right)=\alpha_{j} \cdot \exp \left\{-\frac{\left(D_{j}-D_{0 j}\right)^{2}}{2 w_{j}^{2}}\right\}$

where $f\left(D_{j}\right)$ denotes probability distribution of $j^{\text {th }}$ component of $D$ described by a Gaussian. $A_{j}$ is the fraction of each diffusion component. $\alpha_{j}, w_{j}$, and $D_{0 j}$ are the amplitude, width, and centre of the $j^{\text {th }}$ component of the Gaussian. The $r^{2}$ values of different diffusion modes at different time lags were determined by fitting the CDFs with equation 4 . The mean $D$ values for each diffusion mode were estimated by the slope of the $r^{2}$ plot.

\section{Statistical probability distribution of diffusion coefficient}

HoMOgENEOUS DIFFUSION MODEL. The statistical probability distribution of the diffusion coefficient in a homogeneous environment was calculated using the following equation, ${ }^{33}$

$p(D) \mathrm{d} D=\frac{1}{(N-1) !} \cdot\left(\frac{N}{D_{0}}\right)^{N} \cdot D^{N-1} \cdot \exp \left(\frac{-N D}{D_{0}}\right) \cdot \mathrm{d} D$

where $N$ is the number of the data points in a diffusion trajectory, $D_{0}$ is the mean diffusion coefficient, and $D$ is experimentally obtained diffusion coefficient for an individual trajectory. For this calculation, the experimentally obtained trajectories were all cut such that they contained ten data points (i.e., $N=10$ ). The $D_{0}$ value for the calculation was determined by the CDF analysis of the diffusion data (the $D$ value in equation 1).

GAUSSIAN Distribution MoDel. When the diffusion is described by multiple modes in which the $D$ value distribution is described by a Gaussian, the statistical probability distribution was calculated using the following equation, ${ }^{25}$

$p(D)=\int_{0}^{\infty} f(D) \cdot \frac{1}{(N-1) !} \cdot\left(\frac{N}{D_{0}}\right)^{N} \cdot D^{N-1} \cdot \exp \left(\frac{-N D}{D_{0}}\right) \cdot \mathrm{d} D$

where $f(D)$ denotes probability distribution of $D$ determined by the CDF analysis of the diffusion data using equations 2 and 3. $D_{0}$ was also determined by fitting the CDFs with equations 2 and 3 .

Double Gaussian MODEL. When the diffusion is described by multiple diffusion modes in which the $D$ value distribution is described by multiple Gaussian, the statistical probability distribution was calculated using the following equation,

$p(D)=\sum \int_{0}^{\infty} f\left(D_{j}\right) \cdot \frac{1}{(N-1) !} \cdot\left(\frac{N}{D_{0 j}}\right)^{N} \cdot D_{j}^{N-1} \cdot \exp \left(\frac{-N D_{j}}{D_{0 j}}\right) \cdot \mathrm{d} D_{j}$

where $f\left(D_{j}\right)$ denotes probability distribution of $j^{\text {th }}$ component of $D\left(D_{j}\right)$ determined by the CDF analysis of the diffusion data using equations 4 and 5. $D_{0 j}$ is the mean diffusion coefficient of the $j^{\text {th }}$ component determined by fitting the CDFs with equations 4 and 5 .

\section{Results and Discussion}

Absorption and fluorescence spectra of $\mathbf{1}$ and $\mathbf{2 a}+\mathbf{2 b}$ in THF are virtually identical (Figure 2), with absorption and fluorescence maxima at 523 and $532 \mathrm{~nm}$, respectively. Fluorescence quantum yields of $\mathbf{1}(0.46$ in THF) and $\mathbf{2} \mathbf{a}+\mathbf{2} \mathbf{b}(0.52$ in THF) are comparable to that of a precursor perylene diimide derivative (0.54 in ethanol $)^{23}$. The results confirm that the poly(THF) chains have minimum effect on the fluorescence properties of the fluorophore.

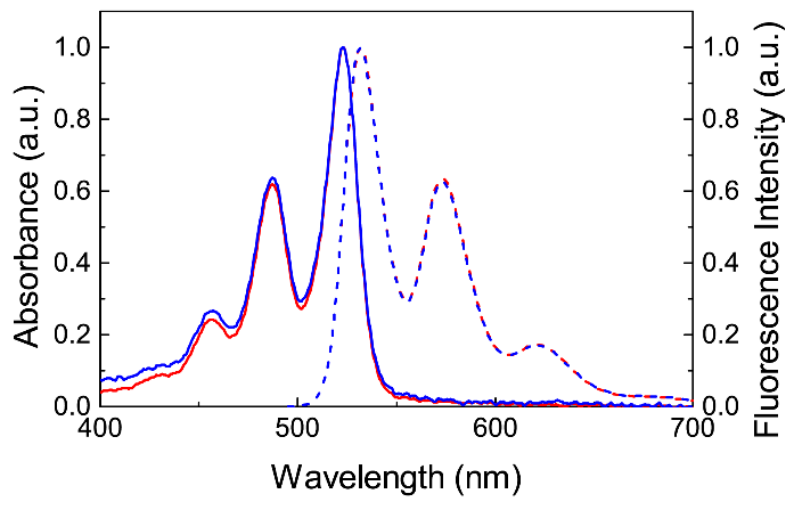

Figure 2. Absorption (solid lines) and fluorescence (broken line) spectra of 1 (red lines) and $\mathbf{2 a} \mathbf{a} \mathbf{2 b}$ (blue lines) in THF. The excitation wavelength of the fluorescence spectra was $488 \mathrm{~nm}$. 
Figure 3 shows single-molecule fluorescence images of $\mathbf{1}$ (Figure 3(a)) and $\mathbf{2 a + 2 b}$ (Figure 3(b)) in the melt of the linear poly(THF) matrix recorded at $303 \mathrm{~K}$. A bright fluorescence of single perylene diimide fluorophore incorporated in the chain was observed which confirmed that the centre-of-mass diffusion of individual polymer molecules in the melt was directly visualized. The spatial locations of the molecule in each image were determined by fitting the image to a twodimensional Gaussian function (see Experimental). Examples of the diffusion trajectories obtained by the image analysis are shown in Figure 3(a) and 3(b) in yellow. The diffusion coefficients of each molecule determined by the MSD analysis of the diffusion trajectories ${ }^{23}$ are plotted in frequency histograms (Figure 4 grey bars). The $D$ distributions obtained in Figure 4 are much broader than those calculated theoretically for a homogeneous diffusion of the polymer chains due to the statistical error of the analysis of the diffusion trajectories (Figure 4 green lines, see Experimental: Statistical probability distribution of diffusion coefficient - homogeneous diffusion model), suggesting heterogeneous diffusion of $\mathbf{1}$ and $\mathbf{2} \mathbf{a}+\mathbf{2 b}$ in the melt state. Similar heterogeneous diffusion has been observed for a linear and mono-cyclic poly (THF) in the melt

(a)
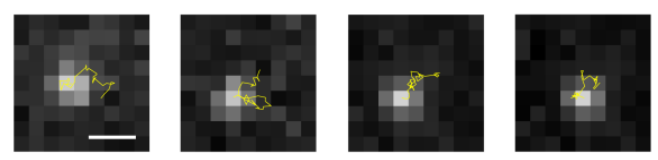

(b)
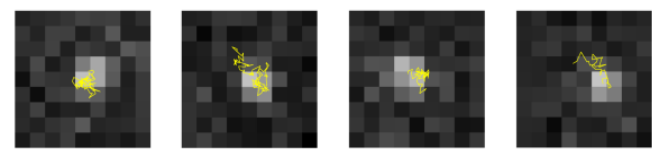

Figure 3. Single-molecule fluorescence images of (a) $\mathbf{1}$ and (b) $\mathbf{2} \mathbf{a}+\mathbf{2} \mathbf{b}$ each mixed with linear poly(THF) melt. The yellow lines show diffusion trajectories. Scale bar $=0.5 \mu \mathrm{m}$.

(a)

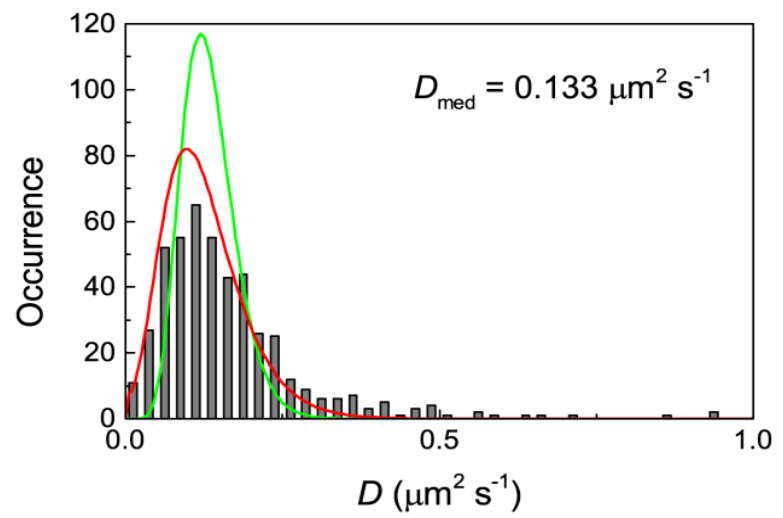

state in the previous study. ${ }^{25}$

The diffusion mode of $\mathbf{1}$ and $\mathbf{2} \mathbf{a}+\mathbf{2 b}$ was further analysed by the cumulative distribution functions (CDFs), $\mathrm{P}\left(r^{2}, i \Delta t\right){ }^{32} \mathrm{~A}$ single-exponential and multi-exponential decaying behaviour of $\mathrm{CDF}$ in the form of 1-P corresponds to a single-mode and multi-mode diffusion, respectively (see Experimental: Cumulative distribution function analysis). The CDFs obtained for $\mathbf{1}$ and $\mathbf{2} \mathbf{a}+\mathbf{2} \mathbf{b}$ show multi-exponential decaying behaviour (Figure 5(a) and 5(b)). The results demonstrate clearly the heterogeneous diffusion of $\mathbf{1}$ and $\mathbf{2} \mathbf{a}+\mathbf{2} \mathbf{b}$ in the melt state. The diffusion of the linear and mono-cyclic poly(THF)s was welldescribed by a broad and continuous distribution of the diffusion coefficient of each polymer chain due to the different numbers of entanglement in each polymer chain. ${ }^{25}$ The CDFs obtained for $\mathbf{1}$ were fitted well by this model (Figure 5(a), see Experimental: Cumulative distribution function analysis Gaussian distribution model). The frequency histogram of the $D$ values obtained from 1 was also reasonably reproduced by the model (Figure 4(a) red line, see Experimental: Statistical probability distribution of diffusion coefficient - Gaussian diffusion model). Furthermore, the $D$ value determined by the CDF coefficients $\left(D=0.125 \mu^{2} \mathrm{~s}^{-1}\right.$, Figure 5(c)) agrees well with the median of the $D$ distribution obtained in the MSD analysis $\left(D=0.133 \mu \mathrm{m}^{2} \mathrm{~s}^{-1}\right.$, Figure 4(a)). All these results strongly suggest that the 4-armed star polymer (1) shows broad and continuous distribution of the diffusion coefficient in the melt state that is described by a Gaussian function.

The mean diffusion coefficient of the 4-armed star polymer (1) in the melt state $\left(D=0.133 \mu \mathrm{m}^{2} \mathrm{~s}^{-1}\right)$ is slower than that of the linear (i.e., 2-armed) polymer $\left(D=0.224 \mu^{2} \mathrm{~s}^{-1}\right)$ with the similar arm length reported previously. Although theoretical description of the diffusion behaviour of those polymers based on the reptation model is not straightforward due to the relatively small numbers of the chain interaction in each arm, the difference in the mean $D$ values can be interpreted by the arm-number dependent diffusion of branched polymers. ${ }^{5}$

(b)

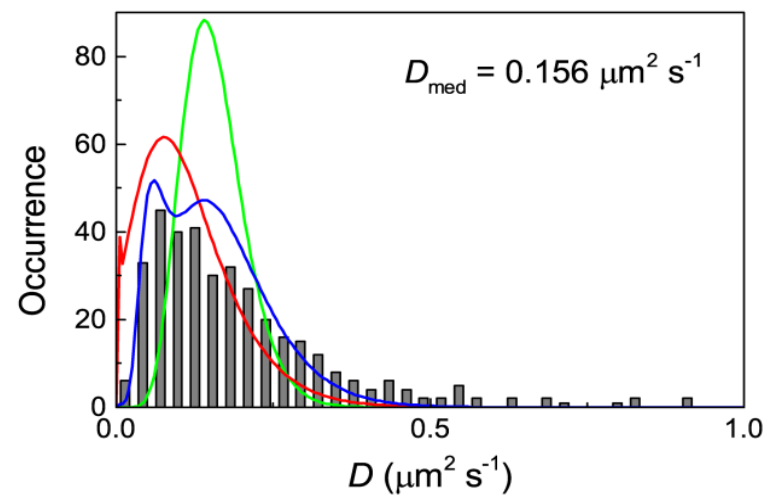

Figure 4. Frequency histograms (grey bars) of the diffusion coefficient determined for (a) $\mathbf{1}$ and for (b) $\mathbf{2 a}+\mathbf{2} \mathbf{b}$ each mixed with linear poly(THF) melt. The solid lines show theoretically calculated probability distributions of the diffusion coefficient based on three different diffusion models; Homogeneous diffusion model (green lines, Eq. 6), Gaussian distribution of the diffusion coefficient (single Gaussian model, red lines, Eq. 7), and two distinct diffusion modes whose distributions are described by Gaussian functions (double Gaussian model, blue line, Eq. 8). The distributions of the diffusion coefficients and the relative amplitudes used in the calculations were determined by the fitting of the cumulative distribution functions with Eq. 1 (homogeneous diffusion model), Eq. 2 (single Gaussian model), and Eq. 4 (double Gaussian model). 
(a)

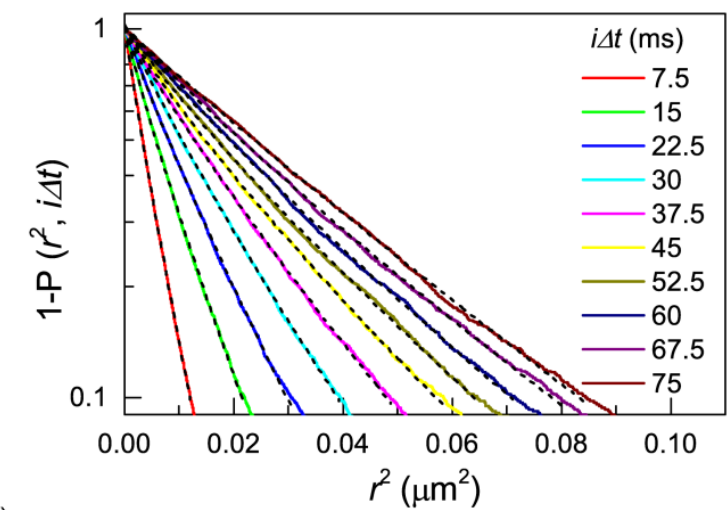

(c)

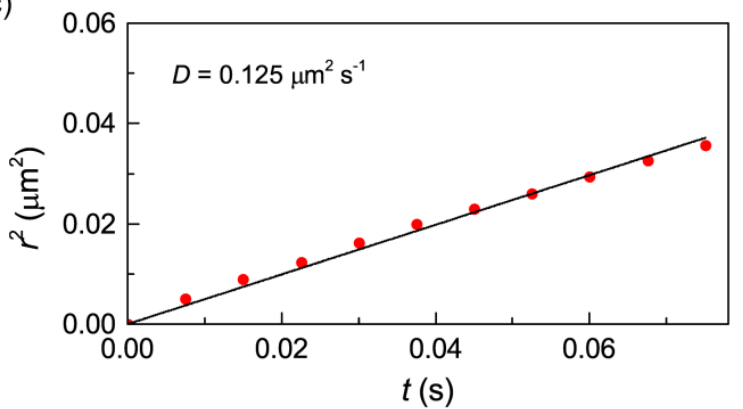

(b)

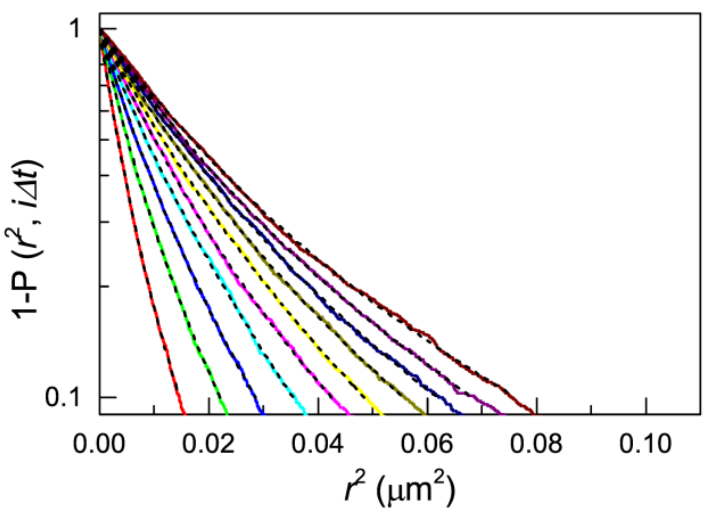

(d)

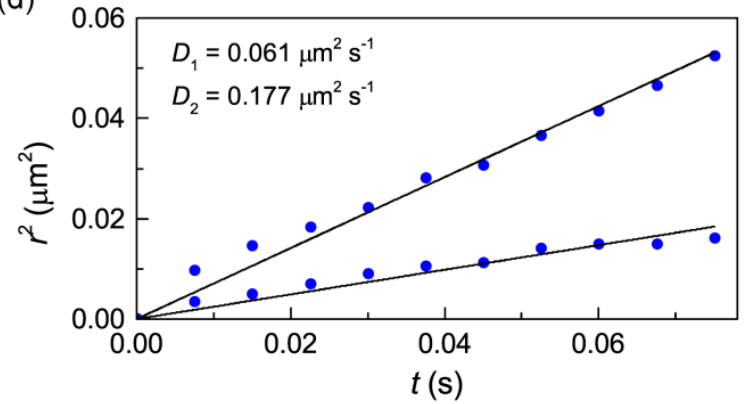

Figure 5. Experimentally obtained cumulative distribution functions ( $i \Delta t=7.5-75 \mathrm{~ms}$ ) in the form of 1-P (solid lines) for (a) $\mathbf{1}$ and for (b) 2a+2b. Dashed lines show fittings with (a) Eq. 2 and (b) Eq. 4. CDF coefficients at different time lags for (c) $\mathbf{1}$ and for (d) $\mathbf{2 a}+\mathbf{2 b}$. The $D$ values for each diffusion mode were determined by either single Gaussian distribution (red) or double Gaussian distribution (blue) models using Eq. 2 and 4 , respectively. The solid lines show linear fittings.

In contrast to the 4-armed star polymer (1), the diffusion mode of the isomeric dicyclic 8-shaped polymer $(\mathbf{2 a}+\mathbf{2 b})$ cannot be described by the single Gaussian distribution model. Although the CDFs were fitted well by the single Gaussian model (see Figure S1), the frequency histogram of the $D$ values showed systematic deviation from the $D$ distribution calculated theoretically based on the CDF analysis (Figure 4(b) red line, see Experimental: Statistical probability distribution of diffusion coefficient - Gaussian diffusion model). Moreover, the $D$ value determined by the CDF coefficients $(D=0.089$ $\mu \mathrm{m}^{2} \mathrm{~s}^{-1}$, Figure S1) does not agree well with the median of the $D$ distribution obtained in the MSD analysis $\left(D=0.156 \mu \mathrm{m}^{2} \mathrm{~s}^{-1}\right.$, Figure 4(b)). These results suggest that the single Gaussian distribution model does not properly describe the diffusion of $\mathbf{2} \mathbf{a}+\mathbf{2} \mathbf{b}$ in the melt state.

Two isomeric forms, vertically oriented (2a) and horizontally oriented (2b) 8-shaped dicyclic polymers, could result in two distinct diffusion modes. Thus, the CDFs obtained from $\mathbf{2 a}+\mathbf{2 b}$ were fitted by a double Gaussian distribution model in which the diffusion is described by two independent broad and continuous distributions (see Experimental: Cumulative distribution function analysis - Double Gaussian model). The CDFs can be fitted well with this model (Figure $5(b)$ ). The frequency histogram of the $D$ values is also reasonably reproduced by the theoretically calculated $D$ distribution based on the model (Figure 4(b) blue line, see Experimental: Calculation of the statistical probability distribution of diffusion coefficient - Double Gaussian model).
Those results strongly suggest the presence of two distinct diffusion modes while we cannot rule out completely the possibility of having more than two modes. Two diffusion coefficients $\left(D=0.061 \mu \mathrm{m}^{2} \mathrm{~s}^{-1}\right.$ and $\left.D=0.177 \mu \mathrm{m}^{2} \mathrm{~s}^{-1}\right)$ were obtained by the linear fits of the CDF coefficients (Figure 5(d) blue circles). The ratio of the fast to slow diffusing components is estimated to be four to one based on the double Gaussian analysis (Figure 4(b)).

Although NMR and SEC measurements on $\mathbf{2 a}+\mathbf{2} \mathbf{b}$ showed no sign of conformational differences between the two isomers, ${ }^{26}$ the single-molecule fluorescence imaging experiment suggests distinct diffusion dynamics of the two topological isomers. As the dimension of the perylene diimide fluorophore unit (1.1 nm length) is nearly a half of the radius of gyration $\left(R_{G}\right)$ of the poly $(\mathrm{THF})$ chain in each ring $\left(R_{G}=2.4 \mathrm{~nm}\right.$, see Supporting Information: Calculation of gyration radius), the chain conformation and the interaction with the linear matrix chains should depend on how the dicyclic chains are connected to the fluorophore while the dicyclic chains of poly(THF) in $\mathbf{2 a}+\mathbf{2 b}$ adopt overall random coil conformation in the both isomers.

The mean $D$ value of the isomeric dicyclic 8-shaped polymer $(2 \mathbf{a}+\mathbf{2 b})$ in the melt state $\left(D=0.156 \mu \mathrm{m}^{2} \mathrm{~s}^{-1}\right)$ is comparable to that of the monocyclic polymer $\left(D=0.155 \mu \mathrm{m}^{2} \mathrm{~s}^{-}\right.$ $\left.{ }^{1}\right)$ with the similar ring size reported previously, which is in sharp contrast to the 4-armed star polymer (1) whose average diffusion coefficient is interpreted as the arm-number dependent diffusion of branched polymers. ${ }^{25}$ Although the 
number average molecular weight of $\mathbf{2} \mathbf{a}+\mathbf{2} \mathbf{b}$ determined by NMR $\left(M_{\mathrm{n}}=12,500\right)$ is much larger than that of the monocyclic polymer $\left(M_{\mathrm{n}}=3,800\right)$, the peak molecular weight of $\mathbf{2 a + 2 \mathbf { b }}$ determined by SEC $\left(M_{\mathrm{p}}=5,600\right)$ is only slightly larger than that of the monocyclic polymer $\left(M_{\mathrm{p}}=4,000\right)$. $\mathbf{2 a}$ and $\mathbf{2 b}$ are structurally similar to $\theta$-shaped and manacle-shaped isomers of a dicyclic polymer. Previous studies demonstrated that the hydrodynamic volume of the $\theta$-shaped isomer is much smaller than that of the manacle-shaped isomer. ${ }^{10,11}$ If the ESA-CF reaction between the poly(THF) and fluorophore occurs at random probability, the molar ratio between $\mathbf{2 a}$ and $\mathbf{2 b}$ should be two to one. This suggests that the major component of the dicyclic 8-shaped polymer (i.e., 2a) should have significantly compact conformation as compared with the monocyclic polymer. The results obtained from the SEC experiments are, therefore, consistent with the predicted contracted conformation of the dicyclic polymer, ${ }^{26}$ which partly explains the small difference in the mean $D$ values between the monocyclic and dicyclic polymers.

The $D$ value of the slower diffusing component observed in $\mathbf{2 a}+\mathbf{2 b}\left(D=0.061 \mu \mathrm{m}^{2} \mathrm{~s}^{-1}\right)$ is much smaller than that in $\mathbf{1}(D=$

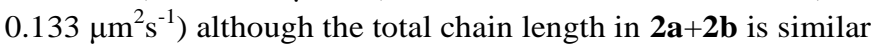
to that in $\mathbf{1}$ (see Supporting Information: Calculation of gyration radius). A similar trend was observed for the monocyclic and linear (i.e., 2-armed) polymers. ${ }^{25}$ The results also coincide with a simulation study which predicts slower diffusion of cyclic chains by the threading with linear chains. ${ }^{34}$ The results indicate efficient threading of the dicyclic chains with the linear matrix chains in one isomer of $\mathbf{2} \mathbf{a}+\mathbf{2} \mathbf{b}$ which results in the slower diffusion of $\mathbf{2} \mathbf{a}+\mathbf{2} \mathbf{b}$ as compared with $\mathbf{1}$. Since the slow diffusing isomer in $\mathbf{2 a}+\mathbf{2 b}$ is a minor component (Figure 4(b)), this component could be assigned to $\mathbf{2 b}$. In contrast to the slower diffusion component, the $D$ value of the faster diffusing component observed in $\mathbf{2 a + 2 b}\left(D=0.17 \mu \mathrm{m}^{2} \mathrm{~s}^{-}\right.$ ${ }^{1}$ ) is slightly faster than that in $\mathbf{1}$. Since the total chain length in $\mathbf{1}$ and in $\mathbf{2} \mathbf{a}+\mathbf{2 b}$ is comparable, the faster diffusion observed for one of the isomer in $\mathbf{2} \mathbf{a}+\mathbf{2} \mathbf{b}$ should reflect less efficient threading of $\mathbf{2} \mathbf{a}+\mathbf{2} \mathbf{b}$ and/or a contracted chain conformation in $\mathbf{2 a}+\mathbf{2 b}$ as compared with the branched counterpart (1), which is suggested by the SEC measurement. Since $\mathbf{2 a}$ is expected to have a contracted chain conformation and represents the major component, this isomer could be responsible for the fast diffusing component. Those results suggest that the chain interaction in the melt state is affected not only by the topological state of the chain but also by the isomeric conformation of the chain.

\section{Conclusion}

In this study, we investigated the diffusion modes of the topological isomers in the polymer melt at the single molecule level. The synthesis of the perylene diimide incorporated dicyclic 8-shaped poly(THF) $(\mathbf{2 a + 2 b})$ provided the unique opportunity to analyse the topological-isomer directed diffusion dynamics. We demonstrated for the first time the distinct diffusion modes of the topological isomers in the melt of the linear matrix polymer by the direct visualization of the diffusive motion of the isomeric dicyclic 8-shaped polymer. Comparisons with the corresponding branched polymer suggested that the two topological isomers were responsible for the two diffusion modes of the dicyclic chain due to their distinct conformational states that lead to isomer-dependent threading with the matrix chains. Such the hidden complexity cannot be revealed by ensemble-averaged techniques. This study clearly demonstrated the importance of visualizing individual chains for the molecular level understanding of the topology and isomer dependent polymer dynamics.

\section{Acknowledgements}

This work was supported by a Grant-in-Aid for Scientific Research No. 22750122 (S.H.), No. 26288099 (T.Y.), and No. 23350050 (Y.T.) of the Japan Society for the Promotion of Science. S.H. is grateful for The Kurata Memorial Hitachi Science and Technology Foundation. The research reported in this publication was supported by the King Abdullah University of Science and Technology (S.H.).

\section{Notes and references}

${ }^{a}$ Biological and Environmental Sciences and Engineering, King Abdullah University of Science and Technology, P.O. Box 4700 KAUST, Thuwal 23955-6900, Kingdom of Saudi Arabia. Email: satoshi.habuchi@kaust.edu.sa

${ }^{b}$ Department of Organic and Polymeric Materials, Tokyo Institute of Technology, 2-12-1 O-okayama, Meguro-ku, Tokyo 152-8552, Japan. Email: ytezuka@o.cc.titech.ac.jp

Electronic Supplementary Information (ESI) available: [calculation of radius of gyration, cumulative distribution function analysis, and movies of single-molecule diffusion]. See DOI: 10.1039/b000000x/

1. P. J. Flory, Principles of Polymer Chemistry, Cornell University Press, 1953

2. P.-G. De Gennes, Scaling Concepts in Polymer Physics, Cornell University Press, 1979.

3. M. Doi and S. F. Edwards, The Theory of Polymer Dynamics, Oxford University Press, 1986.

T. C. B. McLeish, Adv. Phys., 2002, 51, 1379-1527.

M. Rubinstein and R. H. Colby, Polymer Physics, Oxford University Press, 2003.

T. McLeish, Science, 2002, 297, 2005-2006.

7. S. Honda, T. Yamamoto and Y. Tezuka, J. Am. Chem. Soc., 2010, 132, 10251-10253.

S. Honda, T. Yamamoto and Y. Tezuka, Nat. Commun., 2013, 4. K. Heo, Y. Y. Kim, Y. Kitazawa, M. Kim, K. S. Jin, T. Yamamoto and M. Ree, ACS Macro Lett., 2014, 3, 233-239.

10. H. Oike, H. Imaizumi, T. Mouri, Y. Yoshioka, A. Uchibori and Y. Tezuka, J. Am. Chem. Soc., 2000, 122, 9592-9599.

11. Y. Tezuka, A. Tsuchitani, Y. Yoshioka and H. Oike, Macromolecules, 2003, 36, 65-70.

12. J. Klein, Nature, 1978, 271, 143-145.

13. L. Leger, H. Hervet and F. Rondelez, Macromolecules, 1981, 14, 1732-1738.

$14 . \quad$ E. D. Vonmeerwall, E. J. Amis and J. D. Ferry, Macromolecules, 1985, 18, 260-266.

15. A. Deres, G. A. Floudas, K. Mullen, M. Van der Auweraer, F. De Schryver, J. Enderlein, H. Uji-i and J. Hofkens, Macromolecules, 2011, 44, 9703-9709. 
16. S. Habuchi, T. Oba and M. Vacha, Phys. Chem. Chem. Phys., 2011, 13, 6970-6976.

17. H. Aoki, K. Mori and S. Ito, Soft Matter, 2012, 8, 4390-4395.

$18 . \quad$ B. Stempfle, M. Dill, M. J. Winterhalder, K. Mullen and D. Woll, Polym. Chem., 2012, 3, 2456-2463.

$19 . \quad$ B. M. I. Flier, M. C. Baier, J. Huber, K. Mullen, S. Mecking, A Zumbusch and D. Woll, J. Am. Chem. Soc., 2012, 134, 480-488.

$20 . \quad$ C. D. Chapman, S. Shanbhag, D. E. Smith and R. M. RobertsonAnderson, Soft Matter, 2012, 8, 9177-9182.

21. C. Q. Yu, J. Guan, K. J. Chen, S. C. Bae and S. Granick, ACS Nano, 2013, 7, 9735-9742.

22. M. F. Serag, M. Abadi and S. Habuchi, Nat. Commun., 2014, 5 , 5123 .

23. S. Habuchi, N. Satoh, T. Yamamoto, Y. Tezuka and M. Vacha, Angew. Chem. Int. Ed., 2010, 49, 1418-1421.

24. S. Habuchi, in Topological Polymer Chemistry: Progress of Cyclic Polymers in Syntheses, Properties and Functions, ed. Y. Tezuka, World Scientific, Singapore, 2013, pp. 265-290.

25. S. Habuchi, S. Fujiwara, T. Yamamoto, M. Vacha and Y. Tezuka, Anal. Chem., 2013, 85, 7369-7376.

26. S. Fujiwara, T. Yamamoto, Y. Tezuka and S. Habuchi, React. Funct. Polym., 2014, 80, 3-8.

27. K. Adachi, H. Takasugi and Y. Tezuka, Macromolecules, 2006, 39, 5585-5588.

28. T. Yamamoto and Y. Tezuka, Polym. Chem., 2011, 2, 1930-1941.

29. Y. Tezuka and F. Ohashi, Macromol. Rapid Commun., 2005, 26, 608-612.

30. S. Habuchi, S. Onda and M. Vacha, Chem. Commun., 2009, 48684870.

31. S. Habuchi, S. Onda and M. Vacha, Phys. Chem. Chem. Phys., 2011, 13, 1743-1753.

32. G. J. Schutz, H. Schindler and T. Schmidt, Biophys. J., 1997, 73, 1073-1080.

33. M. Vrljic, S. Y. Nishimura, S. Brasselet, W. E. Moerner and H. M. McConnell, Biophys. J., 2002, 83, 2681-2692.

34. Y. B. Yang, Z. Y. Sun, C. L. Fu, L. J. An and Z. G. Wang, J. Chem. Phys., 2010, 133, 064901.

\section{Table of contents entry}

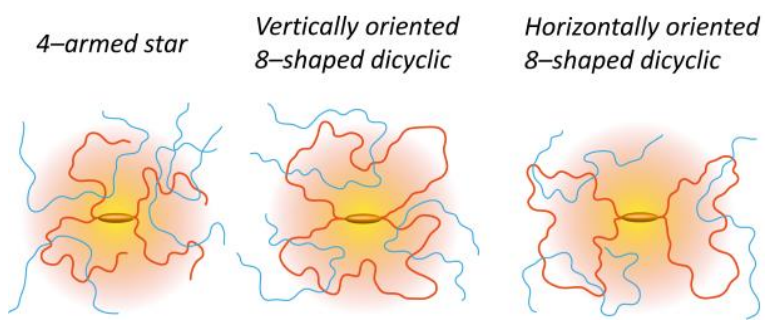

The diffusion modes of the fluorophore incorporated 4-armed star and dicyclic 8-shaped polymers were investigated at the molecular level by means of single-molecule fluorescence imaging. 